\title{
BMJ Open Factors associated with calendar literacy and last menstrual period (LMP) recall: a prospective programmatic implication to maternal health in Bangladesh
}

To cite: Sarker BK, Rahman M, Rahman T, et al. Factors associated with calendar literacy and last menstrual period (LMP) recall: a prospective programmatic implication to maternal health in Bangladesh. BMJ Open 2020;10:e036994. doi:10.1136/ bmjopen-2020-036994

- Prepublication history and supplemental material for this paper is available online. To view these files, please visit the journal online (http://dx.doi. org/10.1136/bmjopen-2020036994).

Received 15 January 2020 Revised 28 September 2020 Accepted 17 November 2020

Check for updates

(C) Author(s) (or their employer(s)) 2020. Re-use permitted under CC BY. Published by BMJ.

For numbered affiliations see end of article.

Correspondence to Mr Bidhan Krishna Sarker; bidhan@icddrb.org

\section{ABSTRACT}

Objective To explore the prevalence and determinants of calendar literacy and last menstrual period (LMP) recall among women in Bangladesh.

Design Cross-sectional survey.

Settings Two rural subdistricts and one urban area from three Northern districts of Bangladesh.

Participants We interviewed 2731 women who had a live birth in the last 1 year.

Primary and secondary outcome measures The primary outcome variable was LMP recall and the secondary outcome was calendar literacy.

Results The majority of participants $(65 \%)$ correctly mentioned the current date according to the English calendar while $12 \%$ mentioned according to the Bengali calendar. During the interview sessions, we used three different calendars: Bengali, English and Hijri to assess calendar literacy. We asked women to mark the current date using the calendar on the day of the interview. Almost $61 \%$ women marked the English calendar, $16 \%$ marked the Bengali calendar and $4 \%$ marked the Hijri calendar correctly. Sixty-three per cent women were found as calendar literate who marked any of the calendars. Among the participants, $58 \%$ had calendars available at their home and only $10 \%$ of women used calendars to track their LMPs. Overall, $53 \%$ women were able to recall their recent LMP. Among the calendar literate, $60 \%$ could recall their LMPs. Factors found associated with recalling LMP were: completed eight or more years of schooling (adj.OR 1.39), primigravida (adj. OR 1.88), the richest wealth quintile (adj.OR 1.55) and calendar literacy (adj.OR 1.59).

Conclusions Despite having reasonable calendar literacy and availability, the use of calendars for tracking LMP found very low. Calendar literacy and sociodemographic characteristics were found as the key factors associated with LMP recall. Maternal, neonatal and child health programmes in low-resource settings can promote a simple tool like calendar and target the communities where ultrasound is not available to ensure accurate LMP recall for early pregnancy registration and timely antenatal care coverage.
Strengths and limitations of this study

- This is the first large-scale study of its kind in Bangladesh, reporting the prevalence of last menstrual period recall in urban and rural settings.

- The study relied on the self-reported data, but the inbuilt quality assurance system minimised the errors and improved the study results' validity.

- We took into account both government and nongovernment health service catchment areas maintaining similar population coverage and statistical rigour.

- We conducted this study in the Northern part of Bangladesh considering higher antenatal care (ANC) coverage, moderate literacy rate and low human resource gaps; hence, the findings are subject to compare contextually.

- Several important factors such as lack of awareness about the importance of gestational age and irregular menstrual cycle were not explored which may influence the risk estimates in this study.

\section{INTRODUCTION}

Every day about 830 women die from pregnancy or childbirth-related complications around the world. Most of these deaths occur in low-resource settings and many of them are preventable. ${ }^{1}$ The South Asia region alone accounts for approximately one-third of the global maternal and child deaths annually. ${ }^{2}$ Globally, the new target set by the Sustainable Development Goals for maternal health is to reduce the mortality ratio less than 70 per 100000 live births by $2030 .{ }^{1}$ According to the recent national data, the maternal mortality ratio in Bangladesh remains unchanged for the last decade (196 deaths per 100000 live births) and a considerable effort is needed to achieve the Sustainable Development Goals. ${ }^{34}$ 
Pregnancy is an important part of women's lives and women should have awareness regarding pregnancy care. ${ }^{5-8}$ Reliable information about the gestational age is crucial to ensure optimal pregnancy care. ${ }^{9}{ }^{10}$ To detect the gestational age, recall of last menstrual period (LMP) plays an important role. Recalling of LMP is also known as the 'calendar method' for predicting the next ovulation date. The calendar method is based on the recognition of cycles in the menstrual period and fertility, in which women record their menstrual cycles. ${ }^{6}$ The WHO also recommends recalling LMP for detecting gestational age because it is a simple and low-cost method. ${ }^{11} 12$ The LMP method has been widely accepted and used in many low and middle-income countries for calculating the gestational age and also a suggested recall method where ultrasound facilities are not readily available. ${ }^{10} 13-15$

Although LMP is used in low-resource settings, including Bangladesh, it is well known that the methods have inherent errors in identifying the LMP date correctly. In most cases, inaccurate LMP recall overestimates the gestational age. ${ }^{12}{ }^{16}$ Furthermore, studies conducted in both developed and developing countries have reported that $15 \%-45 \%$ of women cannot recall their LMPs accurately. ${ }^{12}{ }^{16-18}$ The study has also pointed out that the validity of gestational age calculation with LMP depends on better maternal recall of dates and cycle characteristics, which is somehow related to literacy. ${ }^{14}$

Accurate LMP recall helps to screen for any chance of pre-eclampsia, fetal growth restriction and premature birth outcome. ${ }^{151619-21}$ Accurate LMP recall also enables detecting pregnancy sooner and facilitates early initiation of pregnancy care. Initiation of antenatal care at the right time is crucial because the first 8 weeks of gestational age are very vulnerable for mothers and fetuses. ${ }^{22}$

Calendar literacy can be an integral part of recalling LMP accurately. We carried out this study recognising the knowledge gap and understanding the need to know the underneath factors that expedite the calendar literacy rate and LMP recall. Therefore, this paper aims to explore the prevalence and determinants of calendar literacy and LMP recall in urban and rural areas from three Northern districts in Bangladesh.

\section{MATERIALS AND METHODS}

\section{Study design and settings}

We conducted this cross-sectional study in rural and urban areas in the Northern districts from Bangladesh. The rural sites were located in the Chirirbandar and the Saidpur subdistricts under the districts of Dinajpur and Nilphamary, respectively. The urban site was located in the city corporation of Rajshahi district. According to the population and housing census (2011), Chirirbandar consisted of 142 villages under 12 unions. ${ }^{23}$ The total population of Chirirbandar was 292500 of which 145 881 were women. The average size of the population of each union was 24 375. Saidpur subdistrict consisted of one municipality and five unions with 39 villages. ${ }^{24}$ The total population of Saidpur was 264461 of which 130724 were women. The average size of the population of each union was 27 471. Rajshahi City Corporation consisted of 30 wards. ${ }^{25}$ The total population of the Rajshahi City Corporation was 449756 of which 216782 were women. The average size of the population of each ward was 14 992. According to the census data, the female literacy rate was $42 \%$ in Dinajpur and 39\% for both Nilphamari and Rajshahi districts. ${ }^{26}$ Healthcare utilisation data suggested that at least one antenatal care (ANC) coverage in Dinajpur, Nilphamari and Rajshahi was 75\%, $96 \%$ and $80 \%$, respectively. ${ }^{27}$ Another data showed a medium vacancy $(21 \%-30 \%)$ in terms of healthcare personnel in Dinajpur, Nilphamari and Rajshahi districts. ${ }^{28}$ We considered moderate literacy rate, high ANC coverage and moderate vacancy for human resources to select the study areas.

We considered the area served by the government and non-government community-based health workers as a unit or cluster in rural and urban sites maintaining similar population coverage. The women from each cluster who met the inclusion criteria were invited to participate in the survey. The followings were the inclusion criteria for the study participants' enrolment: (1) had a live birth outcome in the last 1 year, (2) past 28 or more days after the last delivery, (3) could hear, see and speak, (4) had permanent residence in the study area and (5) provided informed consent to interview within 2 days of the first contact. The data collection period was from August to November 2016. In total, 2731 women participated in the study.

\section{Sampling and study participants}

We applied two-stage cluster sampling to select study participants in the study area. In the first stage, we randomly selected 1 subdistrict from 2 districts each and 10 wards (lowest administrative unit of city area) from Rajshahi City Corporation. Then, we randomly selected 6 clusters out of 12 in the Chirirbandar subdistrict, 6 clusters out of 12 clusters in the Saidpur subdistrict and 6 clusters from 10 clusters in Rajshahi. We used a conventional statistical method to calculate the sample size. Based on the selected process indicators and assuming the improvement rate after project implementation, we calculated the sample size for the survey. As this cross-sectional study was conducted to get a baseline status in designing an intervention focusing on the improvement of maternal and child health, we considered the proportions at baseline and end line to estimate the sample size. The sample size was calculated using the formula proposed by Hayes and Bennett. ${ }^{29}$ Considering the proportion of taking any ANC by the first trimester $p_{0}=0.41$ (baseline), $p_{1}=0.57$ (end line) and $\mathrm{K}=0.15$ (coefficient of variation between clusters), we needed the highest number of 150 mothers in each cluster, including 5\% non-response rate. Considering the proportion of taking at least one ANC from medically trained provider $p_{0}=0.49$ and $p_{1}=0.66$ and $\mathrm{K}=0.15$, we needed the highest number of 6 clusters per area to assess the change in the indicators those were 
least known at baseline. For each study area, the sample size was calculated 900 ; the total required sample size was $2700(150 \times 6 \times 3)$.

We recruited study participants through the expanded programme on immunisation method. ${ }^{30}$ At first, we selected the starting point to start data collection in the selected clusters. We consulted with the community people to determine the mid-point of each cluster. We used the bottle spinning method to ensure randomisation. We spun the bottle at the midpoint of each cluster to pick a random direction to start searching study participants. Afterwards, we visited every household on the next door basis as per bottle indicated direction, and interviewers identified eligible participants for interviewing. Data collection continued until the cluster's sample size was met. Though the required sample size was 150 in each cluster but in some cases, the data collection team interviewed one or two more women to cover the entire cluster. Finally, we completed 2731 interviews from the three study areas. If any eligible woman was absent during the household visit, then data collectors tried at least two more times to interview her.

\section{Data collection}

\section{Survey tool development}

We used a semistructured questionnaire to collect data through face-to-face interviews with women. A team of experienced investigators from icddr,b was responsible for developing this survey tool. Based on the research objectives and the expected outcome variables of the study, the research team considered the relevant indicators to design the tool. As part of designing the tool, investigators did the desk review of the relevant literature such as-Bangladesh Demographic and Health Survey, Bangladesh Maternal Mortality and Healthcare Survey and other similar studies. After formulating the tool, we carried out a pretest to check the design of the questionnaire how it works in practice. After addressing all the problems related to the survey tool, the data collection team started collecting data. In the survey tool, we included the basic individual and household characteristics of the study participants (age, religion, years of schooling, employment status, income, household asset and place of residence); reproductive and obstetric information of the participants (age at first marriage, gravida, para, living children, pregnancy history, LMP date), knowledge and practice of calendar literacy (able to mention correctly Bengali and English day, month, year and date which is related to their knowledge; able to mark Bengali/English/Hijri date in the calendar which is related to their practice).

\section{Staff recruitment and training}

We involved a team of efficient and experienced field research assistants for data collection. We recruited field research assistants who had a minimum Bachelor's degree with at least 2 years of previous experience in the relevant field especially in similar positions in large-scale surveys. After recruitment, we trained the interviewers on the survey tool to collect data. During training sessions, the key persons from the research team discussed the objectives of the study so that the data collection team can easily avoid any confusion. Furthermore, we developed a training manual for the interviewers that included interviewing techniques and specific instructions for each of the data collection questionnaire and checklist.

\section{Data management and quality assurance}

A project research physician and a research investigator coordinated the data collection team. Furthermore, we divided the data collection team into three groups. Each group had four data collectors and a group leader. The group leaders were responsible for ensuring quality data regularly. They checked the completeness of every interview on the spot after the data collected through the dayto-day monitoring. Again, as per the monitoring plan, the group leaders and the team coordinators reinterviewed almost one-third of the total interviewees to check the accuracy and the validity of data. We also checked relevant documents (such as calendar) during our data collection to minimise the errors. Expert programmers of the Maternal and Child Health Division of icddr,b designed a database template maintaining skipping options strictly and logically to avoid inconsistency of data. The programming team used Dot net (V.10) software for data template design as appropriate. An expert data management team was engaged in entering all the data through an online database. The data management team simultaneously entered both the precoded and the postcoded data. For postcoding data, the research team was closely involved with the data management team.

\section{Ethics}

icddr,b has rigorous processes to meet research integrity and ethics, which is called the Institutional Review Board (IRB). icddr,b's IRB consists of four mandatory committees involving internal and external topic experts: Research Review Committee (RRC), Ethical Review Committee (ERC), Animal Experimentation Ethics Committee and Programme Coordination Committee. icddr,b attaches great importance to ensuring that all research protocols conducted by its scientists are scientifically sound and meet international ethical standards. To achieve this, in addition, to review at the centre level and by at least two external reviewers, all research protocols are subject to review by at least two of three committees: RRC, ERC (protocols involving human subjects) and the Animal Experimentation Ethics Committee (protocols involving animals).

RRC has the authority to approve, suggest modifications to or disapprove of any proposed research protocol. Any change to an approved research protocol also needs the approval of the RRC. No research protocol can be implemented or proceed for consideration by the ERC or the Animal Experimentation Ethics Committee without the RRC's approval. 
The ERC's approval is required for all protocols involving human subjects. It is an independent body committed to protecting the rights of the people who participate in research protocols conducted under the auspices of icddr,b. A subcommittee of the ERC, the Data Safety Monitoring Board, periodically reviews and evaluates the accumulated study and makes recommendations to the ERC concerning the continuation, modification or termination of the study.

We acquired both RRC and ERC approval from the IRB of icddr,b before we start collecting data. During the data collection period, we acquired written informed consent from all the participants after ensuring that none of them is exposed to minimal risk while participating in this study. Since all our participants were already married by then, we did not need to take consent for the minors from their guardians or parents as per IRB requirements.

\section{Variables and measures}

All the information given by the mothers were selfreported. The primary outcome variable was LMP recall and the secondary outcome was calendar literacy. The rest of the variables were considered as independent variables. The description of all variables is given below-

\begin{tabular}{ll}
\hline List of variables & Explanation \\
\hline $\begin{array}{l}\text { Last menstrual period (LMP) recall } \\
\text { (primary outcome variable) }\end{array}$ & $\begin{array}{l}\text { We considered the first day of LMP. We asked the women about the LMP date of their } \\
\text { menstrual cycle prior to the interview date. }\end{array}$ \\
$\begin{array}{l}\text { Calendar literacy } \\
\text { (secondary outcome variable) }\end{array}$ & $\begin{array}{l}\text { We asked study participants to mark the current date from the three different types of } \\
\text { calendars (Bengali, Hiji and English). If the study participants marked it correctly, we } \\
\text { coded '1' for the correct answer and '0' for the wrong answer. We considered women } \\
\text { as calendar literate if they could accurately mark the current date using any of the } \\
\text { provided calendars. }\end{array}$
\end{tabular}

\begin{tabular}{|c|c|}
\hline $\begin{array}{l}\text { Mentioned the current day, month, year } \\
\text { and date }\end{array}$ & $\begin{array}{l}\text { We asked women whether they could tell the name of the present day, month, year } \\
\text { and the date (the current day of interview) }\end{array}$ \\
\hline Age in years & $\begin{array}{l}\text { We considered completed years of age and categorised into the following ranges } \leq 19 \text {, } \\
20-29, \geq 30 \text { years of age }\end{array}$ \\
\hline Years of schooling & $\begin{array}{l}\text { We considered completed years of schooling and categorised into }<5,5-7 \text { and } \geq 8 \\
\text { years of schooling. }\end{array}$ \\
\hline Place of residence & $\begin{array}{l}\text { We considered interviewing the women with permanent residence at the respective } \\
\text { study sites. We categorised place of residence into rural and urban areas. }\end{array}$ \\
\hline Employment status of the participants & $\begin{array}{l}\text { It consisted of multiple categories such as unemployed, service, business, handicraft, } \\
\text { agriculture, farm/fishing and day labour. }\end{array}$ \\
\hline Gravida & The total number of all pregnancies reported by a woman that she had in her lifetime. \\
\hline Availability of calendar at home & The calendar was available at home during the interview. \\
\hline Purpose of using the calendar & $\begin{array}{l}\text { We asked the study participants about the purposes of using a calendar. Multiple } \\
\text { responses were included such as - calculate days and dates, track the dates of the } \\
\text { menstrual period, remember the special/festival day (Hat day \{weekly shopping day } \\
\text { at village\}/Eid/Puja/Selling certain goods), remember the date of the tuition fee of } \\
\text { children, remember the loan instalment day, child's date of birth and track lunar date. }\end{array}$ \\
\hline Wealth Index & $\begin{array}{l}\text { The Wealth Index was calculated using easy-to-collect data on a household's } \\
\text { ownership of selected assets including primary source of drinking water, boiled water } \\
\text { prior to drinking, the water source for cooking and handwashing, toilet facility, cooking } \\
\text { fuel, main construction material of the roof, the main material of the exterior walls, } \\
\text { the main material of the floor, rooms used for sleeping, ownership of farm animals, } \\
\text { own household, own agricultural land. Besides these, ownership of durable goods } \\
\text { such as - radio, television, mobile phone, refrigerator, shallow machine, computer; } \\
\text { ownership of transport such as - bus, truck, rickshaw, van, motorcycle. The Wealth } \\
\text { Index was a categorical variable that categorised into poorer, poor, middle, rich and } \\
\text { richest. }\end{array}$ \\
\hline
\end{tabular}

\section{Statistical analysis}

We performed a quantitative analysis using the statistical software package STATA, V.13. We used both descriptive and analytical approaches. The study participants' characteristics, including their calendar literacy and the capability of recall LMPs, were presented by mean, median and proportion. To examine the association between outcomes and available covariates, we performed a logistic regression analysis. The potential confounders such as maternal age, place of residence, years of schooling, employment status, and economic condition by asset quintiles were adjusted in the regression analysis. The results were presented by OR with $95 \%$ CI. All statistically significant results were reported at $\mathrm{p}<0.05$.

We generated Wealth Scores by using the principal component analysis method. ${ }^{32}{ }^{33}$ The Wealth Index is a 


\begin{tabular}{lc}
$\begin{array}{l}\text { Table } 1 \text { Sociodemographic characteristics of the survey } \\
\text { participants }\end{array}$ & $\begin{array}{l}\text { Total } \\
\mathbf{n}=2731(\%)\end{array}$ \\
\hline Traits & \\
\hline Age in years & $609(22.30)$ \\
$\leq 19$ & $1693(61.99)$ \\
\hline $20-29$ & $429(15.71)$ \\
$\geq 30$ & \\
Completed years of schooling & $541(19.81)$ \\
\hline$<5$ & $796(29.15)$ \\
\hline $5-7$ & $1394(51.04)$ \\
\hline 8 & \\
Residence & $915(33.50)$ \\
Urban & $1816(66.50)$ \\
\hline Rural & \\
\hline Employment status of the participant & $2583(94.58)$ \\
\hline Unemployed & $148(5.42)$ \\
\hline Employed & \\
Gravida & $1038(38.01)$ \\
\hline 1 & $1693(61.99)$ \\
$\geq 2$ & 2 \\
\hline Median no. of pregnancies (gravida) & $547(20.00)$ \\
\hline Poorer & $546(20.00)$ \\
\hline Poor & $546(20.00)$ \\
\hline Rich & $546(20.00)$ \\
\hline Richest & $20.00)$ \\
\hline
\end{tabular}

${ }^{*}$ Refers to service/business/handicraft/agriculture/farm/fishing, day labour and so on.

composite measure of a household's cumulative living standard. ${ }^{34}$ Information on the Wealth Index is based on the data collected through the household questionnaire. Each household was assigned a standardised score for each asset and the collective score differs from one another depending on whether the household owned the assets or not. These scores are summed up by the availability of the household assets and individuals are ranked according to the total score of the household. The resulting asset scores are standardised concerning a standard normal distribution with a mean of 0 and a SD of 1 . The sample is then divided into population quintiles-five groups with the same number of individuals in each. These standardised scores are then used to create the breakpoints that defined wealth quintiles as: poorer, poor, middle, rich and richest.

\section{RESULTS}

Sociodemographic characteristics of the study participants

Table 1 shows that most of the participants (62\%) belonged to the age group of 20-29 years, followed by
$22 \%$ who were below 20 years of age. Overall, $80 \%$ of women had completed 5 years of schooling of which $51 \%$ had completed eight or more years of schooling. Among the participants, $95 \%$ of women were unemployed. Fifty per cent of the participants mentioned that they had experienced two pregnancies (median 2).

\section{Calendar literacy, use and availability}

Participants who could mention the day, month and year instantly without seeing the calendar

We asked the participants to mention the name of the current day, month and year (on the day of interview) instantly according to the Bengali and the English calendar. Figure 1 shows almost all the participants $(97 \%)$ were able to mention the name of the days as per the Bengali calendar, which was almost the same in both rural and urban areas. On the other hand, $45 \%$ of participants were able to mention the name of days from the English calendar while participants from urban areas could mention better than their rural counterpart (56\% vs $40 \%)$. The majority of the urban women $(77 \%)$ were able to mention the name of the month from the English calendar rather than the name of a month in the Bengali calendar (44\%). More rural women (64\%) mentioned the name of the months from the Bengali calendar compared with the English calendar (54\%). However, the name of the year was mentioned accurately based on the English calendar (urban 85\% vs rural 72\%) whereas only a few participants could name the year from the Bengali calendar (urban $7 \%$ vs rural $6 \%$ ). In case of remembering date, majority participants both in urban and rural areas $(78 \%$ and $58 \%)$ were able to correctly mention the present date based on the English calendar while only $12 \%$ of the total participants mentioned the date from the Bengali calendar.

\section{Capability and purpose of using a calendar}

Table 2 shows that more than half of the women $(61 \%)$ marked the date correctly from the English calendar whereas $16 \%$ marked from the Bengali calendar and only a few of women (4\%) marked from the Hijri calendar. About $63 \%$ of women could mark correctly from any of these three calendars (Bengali, English and Hijri), which we considered as calendar literacy. More than half of the participants $(58 \%)$ had a calendar at their home. About $44 \%$ of women reported using it for calculating days and dates, whereas only $10 \%$ of women used it to track their menstrual cycle.

\section{Calendar literacy and its associated factors}

Association between calendar literacy and sociodemographic characteristics

Online supplemental table 1 indicates that the majority participants $(67 \%)$ from age groups 20-29 were calendar literate. The employed women $(81 \%)$ had more calendar literacy than the unemployed women (62\%). Most of the women $(89 \%)$ who completed eight or more years of schooling were calendar literate. Ninety per cent of the 


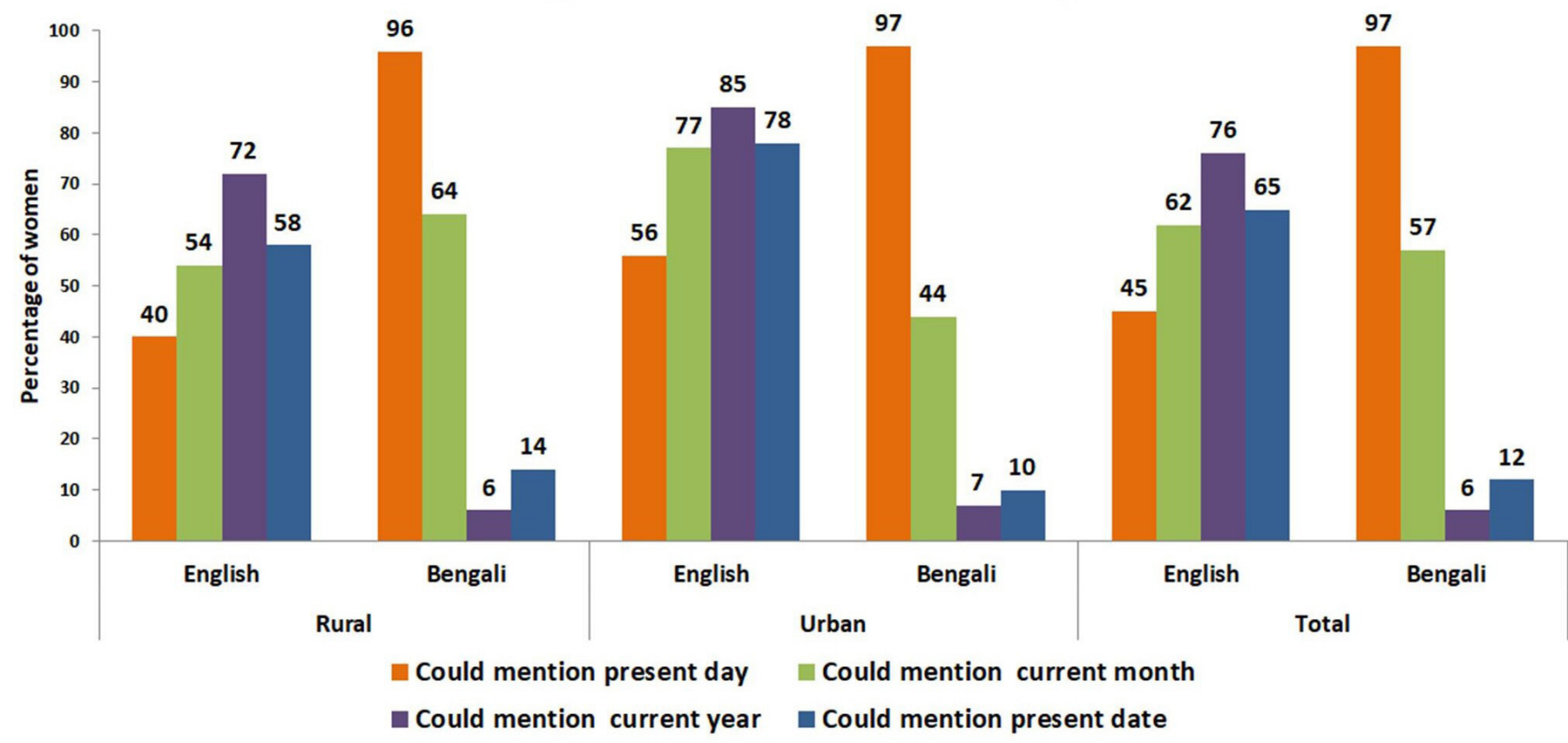

Figure 1 Women who could mention current date.

women who did not complete 5 years of schooling were calendar illiterate.

Educational status of calendar literate women

In figure 2, we explored the relationship between calendar literacy and the educational background of

\begin{tabular}{|c|c|}
\hline Traits & $\begin{array}{l}\text { Total } \mathrm{n}=\mathbf{2 7 3 1} \\
(\%)\end{array}$ \\
\hline Could mark date from the Bengali calendar & $448(16.40)$ \\
\hline Could mark date from the English calendar & $1668(61.08)$ \\
\hline Could mark date from the Hijri calendar & $114(4.17)$ \\
\hline $\begin{array}{l}\text { Could mark date from any of these three } \\
\text { calendars }\end{array}$ & $1730(63.35)$ \\
\hline \multicolumn{2}{|l|}{ Availability of calendar at home } \\
\hline Yes & $1594(58.37)$ \\
\hline No & $1137(41.63)$ \\
\hline \multicolumn{2}{|l|}{ Purpose of using calendar* } \\
\hline To calculate the days and dates & $1204(44.09)$ \\
\hline To track the dates of the menstrual period & $268(9.81)$ \\
\hline $\begin{array}{l}\text { To remember the special/festival day (Hat } \\
\text { day/Eid/Puja/Selling certain goods) }\end{array}$ & 364 (13.33) \\
\hline $\begin{array}{l}\text { To remember the date of the tuition fee of } \\
\text { children }\end{array}$ & $85(3.11)$ \\
\hline To remember the loan instalment day & $61(2.23)$ \\
\hline Others $\dagger$ & 39 (1.43) \\
\hline
\end{tabular}

${ }^{*}$ Refers multiple responses.

†Refers to recall child's date of birth, to calculate the lunar date and so on. the study participants. Seventy-two per cent of women who completed eight or more years of schooling could mark the correct date in the calendar. However, only a few of the women $(3 \%)$ who did not complete 5 years of schooling could also mark the calendar correctly.

\section{Factors associated with calendar literacy}

Online supplemental table 2 presents that almost all the indicators were crudely associated with a higher prevalence of calendar literate women. After adjustment, the OR of calendar literate women who completed eight or more years of schooling was 34 times (adj. OR 34.46, 95\% CI 23.96 to 49.57 ) higher than the women who did not

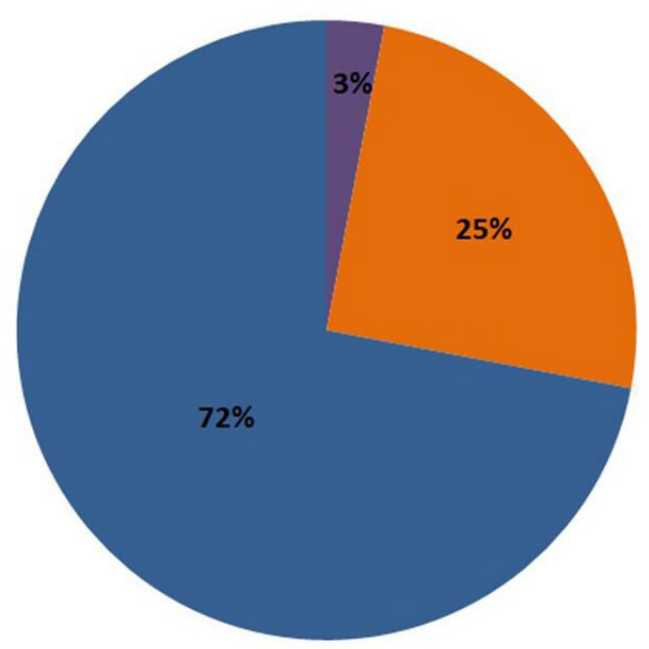

no education or below class $5 \square$ Class 5-7 $\square$ Class 8 and above

Figure 2 Educational status of calendar literate women. 
complete 5 years of schooling. The women who belonged to the top two wealth quintiles (ie, rich adj. OR 2.75, 95\% CI 1.83 to 4.11 and richest adj. OR 3.15 , 95\% CI 1.88 to 5.29) were almost three times more calendar literate than the poorer women.

\section{LMP recall}

Table 3 presents more than half of the participants (53\%) could recall their recent LMP dates. About $59 \%$ of women aged $\leq 19$ years recalled their LMP dates. We found a significant statistical $(p<0.01)$ association between the completed years of schooling and the recent LMP recall. The women who had completed eight or more years of schooling, most of them (60\%) could recall the LMP date. The women who were calendar literate, about $60 \%$ of them could recall their LMPs, and there was a significant $(p<0.01)$ association between the calendar literacy and the recent LMP recall.

\section{LMP recall and its associated factors}

Table 4 present that almost all the indicators were crudely associated with the higher prevalence of recalling LMP. After adjustment, the OR of LMP recall for the women who completed eight or more years of schooling was 1.39 times (adj. OR 1.39, 95\% CI 1.05 to 1.84) higher than the women who did not complete 5 years of schooling. Similarly, the richest women were more likely to recall recent LMP than the poorer women (rich: adj. OR 1.46, 95\% CI 1.08 to 1.97 and richest: 1.55 , 95\% CI 1.09 to 2.21). Besides, the women who had primigravida were more likely to recall recent LMP than those who had multigravida (adj. OR 1.88, 95\% CI 1.55 to 2.29 ). The OR of LMP recall for calendar literate women was 1.59 times (adj. OR 1.59, 95\% CI 1.28 to 1.98) higher than the calendar illiterate women.

The model adjusted for variables such as woman's age, completed years of schooling, residence, employment status, gravida, Wealth Index, availability of calendar at home, the purpose of using a calendar, tracking LMP and calendar literacy.

\section{DISCUSSION}

This study explored the prevalence of LMP recall and calendar literacy along with the factors associated with these two outcome variables. This study suggested that urban women were more calendar literate than their rural counterparts but we did not find any significant difference between these two groups on the prevalence of recalling recent LMP. This study also found that more than half of the women had calendars available at their home; however, only a few women used the calendar to track their LMPs. As expected, calendar literate women could better recall their LMPs than the calendar illiterate women. Besides, years of schooling, fewer pregnancies and socioeconomic class were the significant associated factors for recalling LMP. Women with eight or more years of schooling, first time pregnancy and women from the richest wealth quintile could recall their LMP better.

Because of the unavailability of the studies reporting the prevalence of calendar literacy and the factors associated with calendar literacy, and how calendar literacy contributes to LMP recall we could not compare our findings with any study. We could only compare our findings on the prevalence of LMP recall. However, we discussed the use and importance of calendar method or LMP recall in relation to findings from prior studies around the globe including Bangladesh.

Similar to our findings, a study conducted in 2005 in a developed country found that more than half of the women were capable to recall their LMP accurately. ${ }^{18} \mathrm{~A}$ study conducted in Bangladesh in 2016 examined the significance of LMP recall to measure the gestational age by using the home calendar and explored how it differed from the ultrasound result. The study validated the use of the LMP method for estimating gestational age in a rural setting of Bangladesh. The same study also revealed that a mother's higher education level and previous experience of preterm birth are associated with better accuracy of LMP recall. ${ }^{35}$ Studies also evidenced that LMP is a reliable way to calculate the gestational age while one study had the precision level of $86 \%-90 \%$ for LMP recall's accuracy and reliability. ${ }^{13} 36$

Several studies prioritised the LMP method to determine the gestational age as well as to assess the preterm birth and fetal growth restriction. ${ }^{14}{ }^{37-39}$ A number of studies used the LMP recall method to identify pregnancy and used paper-based calendar as a tool to get the accurate LMP recall and recruited pregnant women immediately after pregnancy confirmation for their surveillance or interventions. ${ }^{12-14} 18$ 35-43 Our cross-sectional survey only explored the use of a calendar, calendar literacy, LMP recall and its associated factors in the area where there is no such paper-based calendar intervention. In our country context, women usually remember LMP dates in an unwritten way. This study found that only $10 \%$ of women used a calendar for tracking LMP. However, in the modern era, everyone seems to be connected to the digital world, with more and more people using calendars inbuilt in their phone, their email or various other options. Many places like homes and businesses still use at least one printed paper calendar. ${ }^{44}$ This study also found that $58 \%$ of households owned a printed calendar and 93\% owned (according to our household asset calculation) at least one cell phone. The printed calendar provides an easy way to see the date without having to $\log$ in and access a tiny screen on the smartphone. So, using a printed calendar to track LMP is still comfortable and relevant for women even in the age of digital technology where an electronic calendar is available on the cell phone.

However, the LMP method has its limitations. LMP can predict gestational age accurately only if the cycle characteristics and the date of onset of the last menstrual bleed can be established. ${ }^{36}$ Despite the limitations, studies 
Table 3 Association between last menstrual period (LMP) recall and sociodemographic characteristics

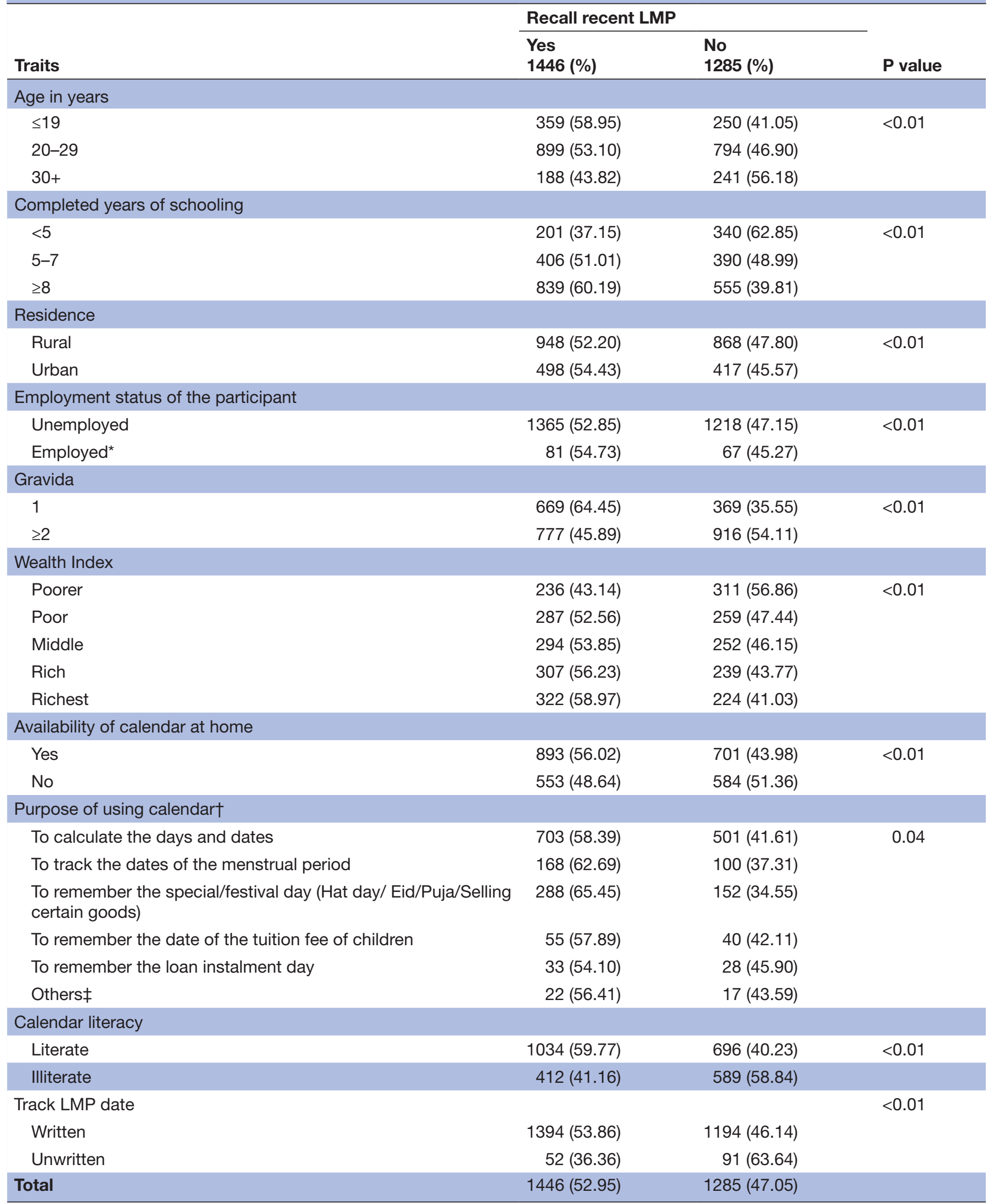

${ }^{*}$ Refers to service/business/handicraft/agriculture/farm/fishing, day labour and so on.

†Refers to multiple responses.

$\ddagger$ Refers to recall child's date of birth, to calculate the lunar date and so on. 
Table 4 Crude and adjusted OR for the factors associated with last menstrual period recall

\section{Covariates}

Constant

The age group of women

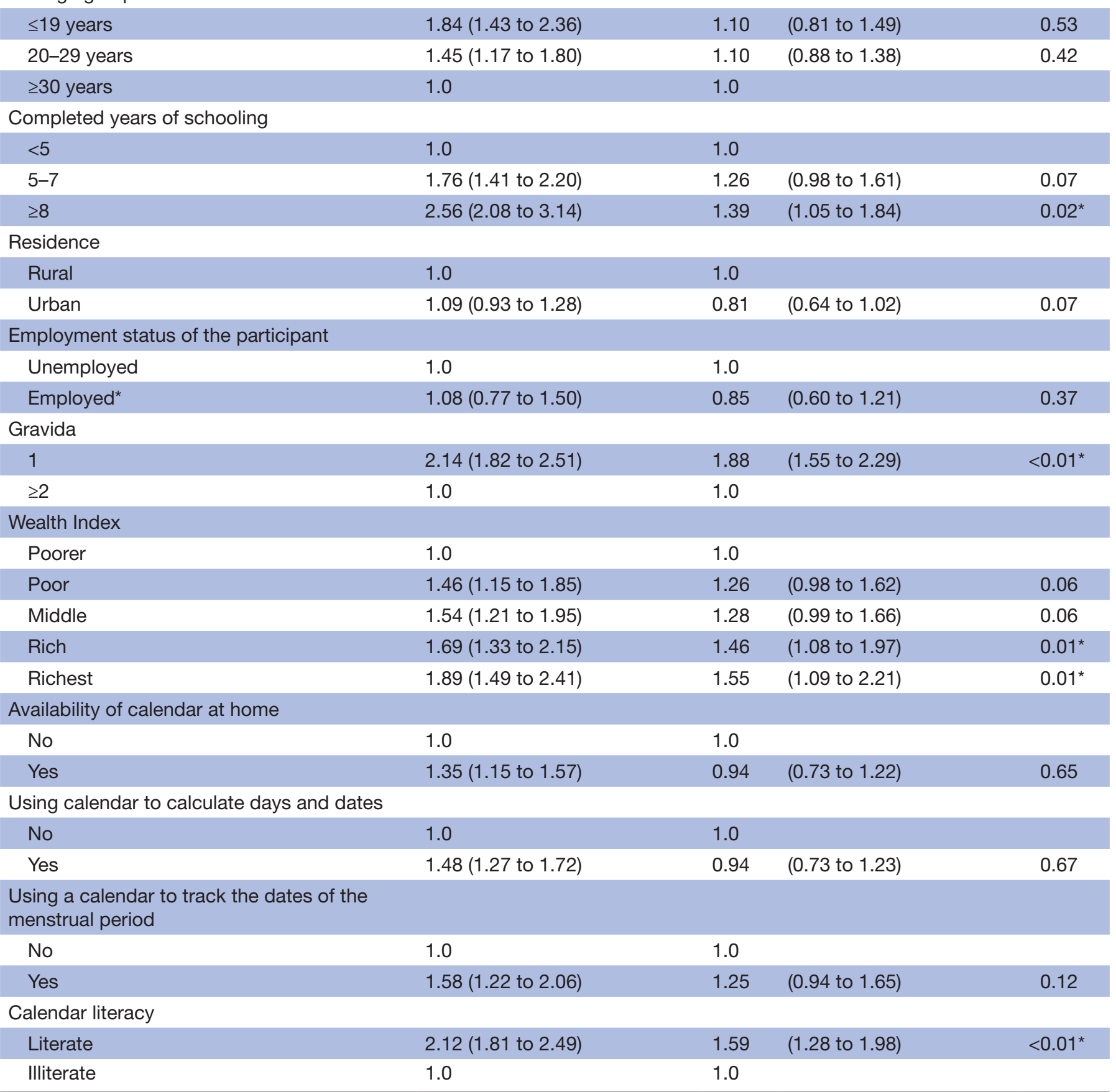

Significant $\mathrm{p} \leq 0.05$.

${ }^{*}$ Refers to service/business/handicraft/agriculture/farm/fishing, day labour and so on. aOR, adjusted OR; cOR, crude OR.

conducted in different geographical settings such as Bangladesh and Guatemala have recommended LMP to be the chosen method for determining gestational age in low-resource settings. ${ }^{124143}$

Since our study was conducted in the Northern part of Bangladesh, where ANC coverage is higher, the literacy rate is moderate and human resource gaps are low; hence, the findings are subject to compare contextually. The analysis was done depending on self-reported information without having a strong surveillance system; therefore, the scope of over or under-reporting may exist. Because of the self-reported data, LMP recall may vary. 
There may be some other confounding factors such as lack of awareness about the importance of gestational age and irregular menstrual cycle were not explored in this study. In Bangladesh, tracking one's menstrual cycle using a calendar is a concept that is yet to become familiar and practised. If women are calendar literate, they will be able to have a clear idea about their menstrual cycle, monitor, and detect any menstrual abnormality. In addition to these, they will be able to calculate the gestational age properly and identify pregnancy at an earlier stage. Early identification of pregnancy can lead to proper and timely utilisation of ANC. This would prevent complications during pregnancy and delivery for the mothers and the babies, thus to improve birth outcomes. We, therefore, feel a high necessity of further nationwide research on this very topic to improve maternal and child health and smoothing the path to achieve Sustainable Development Goals.

\section{CONCLUSION AND RECOMMENDATION}

Accurate recalling of LMP is crucial for maternal health as it helps to calculate the gestational age accurately. It also helps to detect pregnancy early which can influence early initiation of pregnancy care. We found a significant relationship between calendar literacy and LMP recall. Though the calendar literacy rate and the availability of the calendar at home are reasonable; however, the practice of LMP tracking using a calendar is shallow. Besides calendar literacy, factors like years of schooling, place of residence and socioeconomic status have a significant influence on LMP recall too. Mounting all the relevant evidence, we summed up that calendar literacy is an opportunity to introduce a simple tool like paper-based calendar in any maternal, neonatal and child health programmes at communities. And this would be very useful, especially where ultrasound is not available to ensure accurate LMP recall for early pregnancy registration and timely ANC coverage for improving maternal health outcomes in Bangladesh.

\section{Author affiliations}

${ }^{1}$ Maternal and Child Health Division, icddr,b, Dhaka, Bangladesh

${ }^{2}$ School of Health Sciences, Western Sydney University, Greater Western Sydney, New South Wales, Australia

${ }^{3}$ Infectious Diseases Division, icddr,b, Dhaka, Bangladesh

${ }^{4}$ Department of Anthropology, Shahjalal University of Science and Technology, Sylhet, Bangladesh

${ }^{5}$ Department of Public Health, North South University, Dhaka, Bangladesh

${ }^{6}$ Centre of Excellence for Non-Communicable Diseases and Nutrition, James $P$

Grant School of Public Health, BRAC University, Dhaka, Bangladesh

Acknowledgements This research protocol was funded by the Bill \& Melinda Gates Foundation. The awarded BMGF grant number is OPP1146943. icddr,b acknowledges with gratitude the commitment of Bill \& Melinda Gates Foundation to its research efforts. The authors are grateful to their study participants for their spontaneous participation and sincere commitment to fulfil the research endeavour. icddr,b is also grateful to the Governments of Bangladesh, Canada, Sweden and the UK for providing core/unrestricted support.

Contributors BKS, MR, DM, MKM, AA and AR conceived and designed the experiments. BKS, MR, TawR, JJK, MH and MSM performed the experiments. BKS,
TanR, TawR, FA, MH, SNM, AA and AR were involved in analysing data. BKS, MR, TawR, JJK, TanR, MH, FA, MSM and FR contributed to developing the tools. BKS, MR, TanR, TawR, FR, SNM, DM, MKM, AA and AR wrote the paper.

Funding This research protocol was funded by the Bill \& Melinda Gates Foundation. The awarded BMGF grant number is OPP1146943.

Competing interests None declared.

Patient consent for publication Not required.

Provenance and peer review Not commissioned; externally peer reviewed.

Data availability statement Data are available upon reasonable request. Unpublished data are available in accordance with icddr,b's data sharing policy. Requests should be addressed to Ms Armana Ahmend, Head, Research Administration, icddr,b; aahmed@icddrb.org. More information can be found here- https://eur01.safelinks.protection.outlook.com/?url=https $\% 3 A \% 2 F \% 2 F w w w$. icddrb.org\%2Fdmdocuments\%2Ficddrb\%2520Data\%2520Access\%2520Policy. pdf\&data $=02 \% 7 C 01 \% 7$ Canne.laterra\%40care.org\%7C73604d2a 11 bc4750c2 1608d75e12da16\%7Ce83233b748134ff5893ff60f400bfcba\%7C0\%7C1\% 7C637081307376649360\&sdata=fLeMIOsL2d\%2FnkdoDykAoa9kmPxhn3hnJutjwE kOScI4\%3D\&reserved $=0$

Supplemental material This content has been supplied by the author(s). It has not been vetted by BMJ Publishing Group Limited (BMJ) and may not have been peer-reviewed. Any opinions or recommendations discussed are solely those of the author(s) and are not endorsed by BMJ. BMJ disclaims all liability and responsibility arising from any reliance placed on the content. Where the content includes any translated material, BMJ does not warrant the accuracy and reliability of the translations (including but not limited to local regulations, clinical guidelines, terminology, drug names and drug dosages), and is not responsible for any error and/or omissions arising from translation and adaptation or otherwise.

Open access This is an open access article distributed in accordance with the Creative Commons Attribution 4.0 Unported (CC BY 4.0) license, which permits others to copy, redistribute, remix, transform and build upon this work for any purpose, provided the original work is properly cited, a link to the licence is given, and indication of whether changes were made. See: https://creativecommons.org/ licenses/by/4.0\%.

\section{ORCID iDs}

Bidhan Krishna Sarker http://orcid.org/0000-0003-1479-158X

Malay Kanti Mridha http://orcid.org/0000-0001-9226-457X

Anisur Rahman http://orcid.org/0000-0003-1033-5034

\section{REFERENCES}

1 Alkema L, Chou D, Hogan D, et al. Global, regional, and national levels and trends in maternal mortality between 1990 and 2015 with scenario-based projections to 2030: a systematic analysis by the UN maternal mortality estimation Inter-Agency group. Lancet 2016;387:462-74.

2 Wanjira C, Mwangi M, Mathenge E, et al. Delivery practices and associated factors among mothers seeking child welfare services in selected health facilities in Nyandarua South district, Kenya. BMC Public Health 2011;11:360.

3 Streatfield PK, El Arifeen S, Al-Sabir A, et al. Bangladesh maternal mortality and health care survey 2010: summary of key findings and implications. Dhaka, Bangladesh: National Institute of Population Research and Training (NIPORT), 2011.

4 National Institute of Population Research Training, International Centre for Diarrhoeal Disease Research Bangladesh, MEASURE Evaluation. Bangladesh maternal mortality and health care survey 2016: preliminary report: NIPORT, icddr, B, and measure evaluation Dhaka, Bangladesh, and chapel Hill, 2017.

5 Dunson DB, Colombo B, Baird DD. Changes with age in the level and duration of fertility in the menstrual cycle. Hum Reprod 2002;17:1399-403.

6 Sohda S, Suzuki K, Igari I. Relationship between the menstrual cycle and timing of ovulation revealed by new protocols: analysis of data from a self-tracking health APP. J Med Internet Res 2017;19:e391.

7 Stanford JB, White GL, Hatasaka H. Timing intercourse to achieve pregnancy: current evidence. Obstet Gynecol 2002;100:1333-41.

8 Wilcox AJ, Weinberg CR, Baird DD. Timing of sexual intercourse in relation to ovulation. Effects on the probability of conception, survival of the pregnancy, and sex of the baby. $N$ Engl J Med 1995;333:1517-21. 
9 Tunón K, Eik-Nes SH, Grøttum P. A comparison between ultrasound and a reliable last menstrual period as predictors of the day of delivery in 15,000 examinations. Ultrasound Obstet Gynecol 1996;8:178-85.

10 Ghani A, Nahar A, Sultana N, et al. Prediction of gestational age by last menstrual period (LMP) in comparison to ultrasonography (USG). Journal of Shaheed Suhrawardy Medical College 2014;6:82-6.

11 World Health Organization. Home-based maternal records: guidelines for development, adaptation and evaluation. World Health Organization, 1994.

12 Salam SS, Ali NB, Rahman AE, et al. Study protocol of a 4- parallel arm, superiority, community based cluster randomized controlled trial comparing paper and e-platform based interventions to improve accuracy of recall of last menstrual period (LMP) dates in rural Bangladesh. BMC Public Health 2018;18:1-8.

13 Rosenberg RE, Ahmed ANU, Ahmed S, et al. Determining gestational age in a low-resource setting: validity of last menstrual period. Journal of health, population, and nutrition 2009;27:332.

14 Karl S, Li Wai Suen CSN, Unger HW, et al. Preterm or not--an evaluation of estimates of gestational age in a cohort of women from Rural Papua New Guinea. PLoS One 2015;10:e0124286.

15 Macaulay S, Buchmann EJ, Dunger DB, et al. Reliability and validity of last menstrual period for gestational age estimation in a low-tomiddle-income setting. J Obstet Gynaecol Res 2019;45:217-25.

16 van Oppenraaij RHF, Eilers PHC, Willemsen SP, et al. Determinants of number-specific recall error of last menstrual period: a retrospective cohort study. BJOG 2015;122:835-41.

17 Waller DK, Spears WD, Gu Y, et al. Assessing number-specific error in the recall of onset of last menstrual period. Paediatr Perinat Epidemiol 2000;14:263-7.

18 Wegienka G, Baird DD. A comparison of recalled date of last menstrual period with prospectively recorded dates. J Womens Health 2005;14:248-52

19 Kamana K, Shakya S, Zhang H. Gestational diabetes mellitus and macrosomia: a literature review. Annals of Nutrition and Metabolism 2015;66:14-20.

20 Cnattingius S, Villamor E, Lagerros YT, et al. High birth weight and obesity - a vicious circle across generations. Int $J$ Obes 2012;36:1320-4.

21 Salahuddin M, Pérez A, Ranjit N, et al. Predictors of severe obesity in low-income, predominantly Hispanic/Latino children: the Texas childhood obesity research demonstration study. Prev Chronic Dis 2017;14:E141.

22 Poma PA. Early detection of pregnancy. Journal of the National Medical Association 1984;76:305.

23 Bangladesh Bureau of Statistics, Statistics and Informatics Division, Ministry of Planning. Bangladesh population and housing census 2011, community report, Dinajpur: Bangladesh Bureau of statistics (BBS), statistics and informatics division (SID), Ministry of planning, 2014.

24 Bangladesh Bureau of Statistics, Statistics and Informatics Division, Ministry of Planning. Population and housing census, community report, Nilphamari: Bangladesh Bureau of statistics (BBS), statistics and informatics division (SID), Ministry of planning, 2013.

25 Bangladesh Bureau of Statistics, Statistics and Informatics Division, Ministry of Planning. District statistics 2011, Rajshahi: Bangladesh Bureau of statistics (BBS), statistics and informatics division (SID), Ministry of planning, 2013.
26 Bangladesh Bureau of Statistics, Statistics and Informatics Division,, Ministry of Planning. Population \& Housing Census 2011: Bangladesh Bureau of Statistics (BBS), Statistics and Informatics Division (SID) and Ministry of Planning, 2015.

27 MEASURE Evaluation, ICDDR B. Bangladesh District level sociodemographic and health care utilization indicators: National Institute of Population Research and Training (NIPORT), MEASURE Evaluation, UNC-CH, USA and ICDDR, $B, 2011$.

28 Human Resource management, Ministry of Health and Family Welfare. HRH data sheet 2014: human resource management (HRM), Ministry of health and family welfare (MOHFW), Bangladesh Secretariat, Dhaka, 2014.

29 Hayes RJ, Bennett S. Simple sample size calculation for clusterrandomized trials. Int J Epidemiol 1999;28:319-26.

30 Bostoen K, Chalabi Z. Optimization of household survey sampling without sample frames. Int J Epidemiol 2006;35:751-5.

31 Myatt M. A short guide to undertaking surveys using the simple spatial survey method (S3M), 2012

32 Vyas S, Kumaranayake L. Constructing socio-economic status indices: how to use principal components analysis. Health Policy Plan 2006;21:459-68.

33 Wold S, Esbensen K, Geladi P. Principal component analysis. Chemometrics and Intelligent Laboratory Systems 1987;2:37-52.

34 United States Agency for International Development (USAID). The DHS program 2020. Available: https://dhsprogram.com/topics/ wealth-index/ [Accessed 6 Sep, 2020].

35 Shah R, Mullany LC, Darmstadt GL, et al. Incidence and risk factors of preterm birth in a rural Bangladeshi cohort. BMC Pediatr 2014;14:112.

36 Ellertson C, Elul B, Ambardekar S, et al. Accuracy of assessment of pregnancy duration by women seeking early abortions. Lancet 2000;355:877-81.

37 Rashid H, Ma E, Ferdous F, et al. First-Trimester fetal growth restriction and the occurrence of miscarriage in rural Bangladesh: a prospective cohort study. PLoS One 2017;12:e0181967.

38 Baqui $\mathrm{AH}$, Rosen HE, Lee ACC, et al. Preterm birth and neonatal mortality in a rural Bangladeshi cohort: implications for health programs. J Perinatol 2013;33:977-81.

39 Lee AC, Mullany LC, Ladhani K, et al. Validity of newborn clinical assessment to determine gestational age in Bangladesh. Pediatrics 2016;138:e20153303.

40 Unger $\mathrm{H}$, Thriemer $\mathrm{K}$, Ley $\mathrm{B}$, et al. The assessment of gestational age: a comparison of different methods from a malaria pregnancy cohort in sub-Saharan Africa. BMC Pregnancy Childbirth 2019;19:1-9.

41 LÅ P, Arifeen S, Ekström E-C, et al. Effects of prenatal micronutrient and early food supplementation on maternal hemoglobin, birth weight, and infant mortality among children in Bangladesh: the MINIMat randomized trial. Jama 2012;307:2050-9.

42 Gernand AD, Paul RR, Ullah B, et al. A home calendar and recall method of last menstrual period for estimating gestational age in rural Bangladesh: a validation study. J Health Popul Nutr 2016;35:34.

43 Neufeld LM, Haas JD, Grajéda R, et al. Last menstrual period provides the best estimate of gestation length for women in rural Guatemala. Paediatr Perinat Epidemiol 2006;20:290-8.

44 VIIKING. Why printed calendars are still relevant in the digital age. Available: https://mrshirt.com/printed-calendars-are-still-relevant [Accessed 8 July 2020]. 